\title{
Efficacy of modified complex decongestive therapy on limb girth, skin thickness, and functional capacity in patients with lower limb secondary lymphoedema
}

\author{
DOI: https://doi.org/10.5114/pq.2020.100278
}

\author{
Fatma Aboelmagd M., Ashraf Abdelaal Mohamed Abdelaal \\ Department of Physical Therapy for Cardiovascular/Respiratory Disorders and Geriatrics, Faculty of Physical Therapy, \\ Cairo University, Giza, Egypt
}

Abstract

Introduction. Lymphoedema is a serious health problem that impairs functional performance. The study aim was to explore the effects of modified complex decongestive therapy (MCDT) on lower limb oedema, skin layer thickness, and functional capacity in patients with unilateral lower limb secondary lymphoedema.

Methods. Overall, 49 patients (30 women and 19 men) with unilateral lower limb secondary lymphoedema were divided into the MCDT group and the traditional physical therapy (TPT) group and received the assigned treatment for 8 weeks. Limb girth and skin layer thickness at 4 levels and functional capacity were measured before and after the programs with limb round measurements, ultrasonography, and the 6-minute walking test, respectively.

Results. The oedema significantly decreased at the levels of 5 th metatarsal head, medial malleolus, $20 \mathrm{~cm}$ below, and $20 \mathrm{~cm}$ above the patella by $19.2 \%, 28.34 \%, 16.57 \%$, and $12.08 \%$ in the MCDT group and by $13.47 \%, 15.27 \%, 8.78 \%$, and $7.15 \%$ in the TPT group. The skin thickness decreased significantly at the levels of foot, medial malleolus, mid-calf, and mid-thigh by $70.32 \%$, $63.32 \%, 31.82 \%$, and $15.46 \%$ in the MCDT group and by $54.4 \%, 37.26 \%, 21.02 \%$, and $9.12 \%$ in the TPT group. The 6 -minute walking test score increased significantly by $9.84 \%$ in the MCDT group and by $5.52 \%$ in the TPT group. The post-study results comparison of the 2 groups revealed statistically significant differences in all outcome measures $(p>0.05)$ in favour of the MCDT group.

Conclusions. MCDT yielded higher significantly favourable effects than TPT in patients with lower limb secondary lymphoedema. Key words: lymphoedema, modified complex decongestive therapy, oedema, skin thickness, functional capacity

\section{Introduction}

Lymphoedema is a serious chronic health problem characterized by impaired lymphatic system function that leads to accumulation of the lymphatic fluid within the tissues [1]. Lymphoedema, affecting millions of patients globally, is commonly misdiagnosed and incorrectly managed [1, 2]. It occurs in females more often than in males and is usually presented in a progressive unilateral, distal to proximal pattern. Secondary lymphoedema is a slowly progressive disorder that harmfully affects the subject's physical, as well as psychological functions [3], starting with leg pains and aching, diminished walking ability and participation in social events, chronic discomfort, deformity, easy fatigability [4], impaired ability to perform daily functional activities, deteriorated quality of life, reduced self-esteem and body image [5].

Dependency on ambulation makes the lower limb lymphoedema more severely debilitating than upper limb one [3], especially when considering the progressive nature of the protein-rich fluid stagnation that characterizes lower limb lymphoedema [6]. Although there is no cure for lymphoedema at this time, effective treatment procedures are available [7], usually using classical massage techniques, limb positioning, and electrotherapy modalities [8]. In spite of the growing attention towards lymphoedema, the affected subjects are still in need for more care [9], and adequate treatment is required to reduce the lymphoedema-associated physical, psychological, and economical burdens [7].
Despite the recent advance in lymphoedema management, the effectiveness of many therapeutic procedures is still underestimated; this may be in part due to a limited number of high quality studies in this field [10]. The frequently described therapeutic regimens usually consist of 2 phases: the initial acute phase, which typically involves complex decongestive therapy (CDT) [11], and the second phase - a selfdirected maintenance phase that emphasizes the patient's education on self-care and adherence to the therapeutic regimen to obtain the required treatment outcomes [12].

CDT is an effective approach utilized in the treatment of lymphoedema [13]; it enhances the normal lymph flow [14]. CDT stands as a cornerstone in the treatment of lymphoedema because of its desirable effects on the limb volume and quality of life [15]. It combines manual lymph drainage, bandaging, exercise therapy, and skin care [6, 11].

In spite of the recent progress in lymphoedema treatment, further studies are still required to provide sufficient data regarding the efficiency of CDT in the treatment of lymphoedema [14] and to explore short- and long-term effects of CDT in patients with lower limb secondary lymphoedema (LLSL) [16]. Therefore, the objective of the current study was to explore the effects of the modified CDT (MCDT) technique on lower limb oedema, skin layer thickness, and functional capacity in patients with unilateral LLSL.

Correspondence address: Fatma Aboelmagd M., Department of Physical Therapy for Cardiovascular/Respiratory Disorders and Geriatrics, Faculty of Physical Therapy, Cairo University, 7 Ahmed Elzaiat St., Ben Elsaryat, El Dokki, Giza, Egypt, e-mail: fatma.aboelmagd@pt.cu.edu.eg

Received: 29.03.2020

Accepted: 22.04.2020

Citation: Aboelmagd FM, Abdelaal AAM. Efficacy of modified complex decongestive therapy on limb girth, skin thickness, and functional capacity in patients with lower limb secondary lymphoedema. Physiother Quart. 2021;29(2):49-55; doi: https://doi.org/10.5114/pq.2020.100278. 


\section{Subjects and methods}

\section{Study design}

This study was designed as a randomized controlled trial. The clinical part of the study was conducted between April 2016 and November 2017.

\section{Subjects}

A total of 49 volunteer patients ( 30 women and 19 men) with unilateral $(67.35 \%$ right, $32.65 \%$ left) LLSL were recruited from Kasr El Ainy Hospital, Egypt to be enrolled in this study. Included were patients aged 40-60 years, ambulant, with established unilateral LLSL (stage II, III) in accordance with the International Society of Lymphology guidelines [1]. The predetermined exclusion criteria were the following: primary lymphoedema, previous lymphatic reconstructive or debulking procedures, CDT within the previous 6 months, acute inflammation, deep venous thrombosis, active infections, exercise-limiting cardiovascular or neuromuscular diseases, congestive heart failure, chronic kidney diseases, liver diseases, hypertension, or history of psychological or psychiatric disturbances. All patients were informed in detail about the study purpose, risks, and procedures.

\section{Randomization}

After medical counselling, each patient was randomly assigned to either of the 2 groups: the traditional physical therapy (TPT) group or the MCDT group, by opening an opaque envelope containing a randomly generated number. The Research Randomizer software (https://www.randomizer.org) was used in the process of generating random numbers (in which the desired number of sets/groups was 2 and the requested number of patients per each set was specified as 25 , with the total patient number of 50 ) to allocate patients into either TPT treatment $(n=25)$ or the MCDT group $(n=24)$, as shown in Figure 1.

\section{Assessment procedures}

The assessment process began after the first contact with the patient, in which the physical therapy evaluation sheet was filled in with individual patient data, including: personal data, data about lymphoedema (cause, year of diagnosis, medications taken), vital signs, and physical examination of cardiac and pulmonary systems. All patients underwent the same evaluation procedures before starting and after finishing the treatment program. The lower limb oedema girth $(\mathrm{cm})$, skin layer thickness $(\mathrm{mm})$, and functional capacity $(\mathrm{m})$ (6-minute walking test [6MWT]) were the main evaluated pre- and

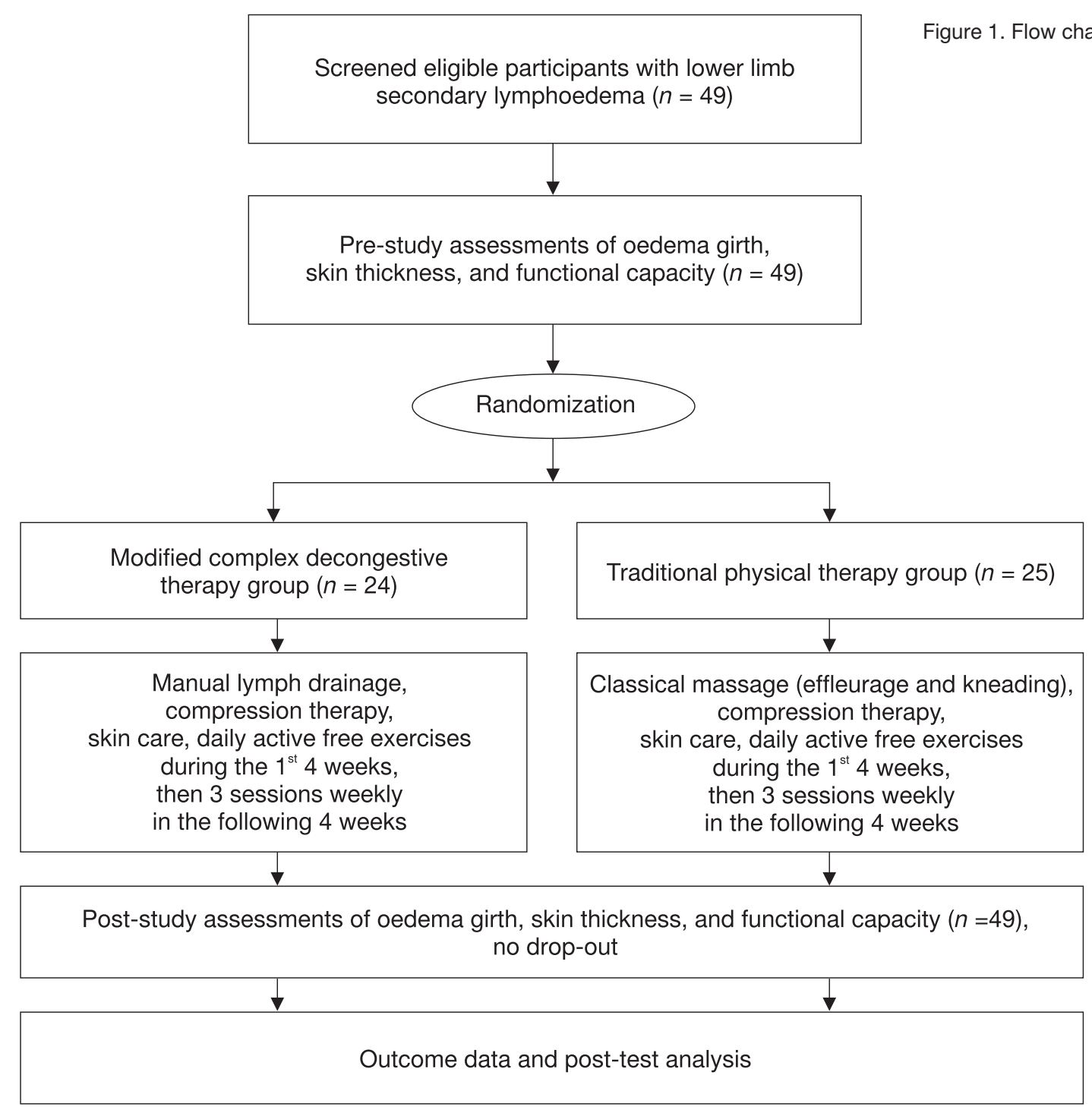


post-study outcomes. The assessors were blind to the patients' group allocation and treatment regimen throughout the study. The therapists were blind to the assessment process and outcome measures throughout the study. Each patient was closely monitored for any alarming signs throughout the study. For patient safety, it was planned to terminate the evaluation or treatment session if blood pressure reached $200 / 110 \mathrm{~mm} \mathrm{Hg}$ or heart rate reached $80 \%$ of predicted maximum heart rate $(220$ - age) [17].

\section{Assessment of the oedema (limb girth)}

Lower limb circumference $(\mathrm{cm})$ was evaluated by the same assessor in all patients by using a flexible, plastic, non-stretchable tape, in accordance with the previously published guidelines [18], at 4 anatomical levels: the $5^{\text {th }}$ metatarsal head (the dorsum of the foot), medial malleoli, $20 \mathrm{~cm}$ below, and $20 \mathrm{~cm}$ above the superior pole of the patella.

\section{Assessment of the skin layer thickness}

Skin and subcutaneous tissue layer thickness $(\mathrm{mm})$ for all patients was scanned with ultrasonography (GE Voluson Pro, high-frequency $22 \mathrm{MHz}$; China) by a single examiner following the same scanning protocol. After a 10-minute rest in a relaxed horizontal position, the lower limb was scanned at 4 anatomical sites along the medial aspect of the lower limb: the $5^{\text {th }}$ metatarsal head (the foot level), medial to medial malleolus, medial to mid-calf, and mid-inner side of the thigh [19].

\section{Assessment of the functional capacity (6MWT)}

The 6MWT was performed in accordance with the previously published guidelines [20] in a $40-\mathrm{m}$ flat corridor. The patient was asked to walk freely in their maximum pace for 6 minutes, without running along the corridor. Heart rate and blood pressure were monitored (via a Panasonic EW3109W portable upper arm blood pressure monitor; North America). The distance $(\mathrm{m})$ covered in the 6-minute walk was then recorded.

\section{Treatment programs}

All patients were encouraged to adhere to their drug therapy, as well as maintain regular diet and normal daily activities throughout the study. To ensure full patient adherence to the given instructions at home, as it is a major point of concern for treatment success [1], weekly plan reports were regularly delivered and checked from patients' relatives who served as supervisors at home. There were no adverse events or remarks during the study. All participants adhered to the prescribed treatment; no drop-out or absence was observed throughout the study. The treatment programs in both groups consisted of 1-hour daily sessions for 4 successive weeks followed by another 4 weeks in which the treatment was applied in 3 sessions/week.

\section{Modified complex decongestive therapy group}

Each patient in the MCDT group $(n=24)$ received a welldesigned CDT program in accordance with the previously described procedures [18] for about 1 hour. MCDT was composed of manual lymph drainage (MLD), compression therapy (using short-stretch compression bandages for first 4 successive weeks, then applying the LegAssistTH, a custom-fit garment, during the last 4 weeks), skin care, and free active exercises. The MLD followed the previously described procedures, in which MLD decongestion sessions progress through cervical, axillary, superficial and deep abdominal, femoral, leg, and foot lymph nodes stimulation [21]. It was started with firm pressure resorptive (strokes) techniques proceeding from proximal to distal regions; the drainage stroke force took an upward, distal-to-proximal application direction and was followed by a gentle sweeping pressure and fluid mobilization technique [18].

After proper cleaning, drying, moisturizing, and padding of the skin, the compression therapy was started using the short stretch bandages during the first 4 successive weeks, in which the layers and overlaps are more in distal end than the proximal end. During the last 4 weeks of the study, the non-elastic containment LegAssistTH system was applied instead of the multilayer bandaging. The LegAssistTH is a pressure applying garment with circumferential Velcro straps extending from the ankle till a point high in the thigh, providing up to $40 \mathrm{~mm} \mathrm{Hg}$ compressive forces and recommended for treatment of lymphoedema [22]. Patients were instructed to wear the compression garment all over the day and perform the active exercise program while wearing it. Exercise training involved deep diaphragmatic breathing (3-5 times), active hip, knee, ankle, and toe free exercises in all directions (10 repetitions each), 5-minute exercise on a stationary bicycle (90 rpm), and, finally, walking on a treadmill (1.6 mph velocity for 5 minutes). Education on the self-administered MLD, skin care (properly inspecting, cleaning, and moisturizing), and exercise training while wearing the compression garment at home was also emphasized.

\section{Traditional physical therapy group}

The TPT program included classical lower limb massage (effleurage and kneading) started from distal to proximal, compression therapy (typically as described for the MCDT group), skin care, and free active exercise while wearing a compression garment as described for the MCDT group. The patients in the TPT group $(n=25)$ were also educated and instructed to adhere to the classical massage techniques, skin care, compression garment wearing, and free active exercise at home.

\section{Statistical analysis}

Statistical analyses were performed by using the SPSS software (version 16.0; SPSS Inc., Chicago, USA). The normality of data was initially tested via the Kolmogorov-Smirnov test and the data showed parametric distribution. Descriptive statistical analysis was done to express the study variables as mean $\pm S D$. Mean changes in the limb girth, skin thickness, and functional capacity within each group were analysed with a paired $t$-test. Between-group differences were analysed with an unpaired $t$-test. Percent of changes (\%) in the evaluated variables were calculated for each group. The significance level for all tests was set at $p<0.05$.

\section{Ethical approval}

The research related to human use has complied with all the relevant national regulations and institutional policies, has followed the tenets of the Declaration of Helsinki, and has been approved by the institutional review board at Faculty of Physical Therapy, Cairo University (approval No.: P.T.REC/ 012/002123). 


\section{Informed consent}

Informed consent has been obtained from all individuals included in this study.

\section{Results}

Statistically non-significant differences $(p>0.05)$ were found between the 2 groups (MCDT and TPT) at baseline with regard to the demographic data (age, height, weight, and body mass index), as well as all the outcome measures (Table 1).

Within-group comparison of the limb girth and the skin thickness revealed a significant decrease at the 4 levels of measurement $(p<0.05)$ and also a statistically significant increase in functional capacity $(p<0.05)$ at the end of the study (Table 2).

The between-group comparison revealed statistically significant post-study differences in the mean values of lower limb girth at the level of $5^{\text {th }}$ metatarsal head $(p=0.004)$, medial malleolus ( $p=0.007), 20 \mathrm{~cm}$ below the patella $(p=0.03)$, and $20 \mathrm{~cm}$ above the patella $(p=0.02)$ in favour of the MCDT group (Figure 2).

Also, post-study statistically significant differences were found between the 2 groups, in favour of the MCDT group, in mean values of lower limb skin thickness at the foot level $\left(p=5.22^{-12}\right)$, medial to medial malleolus level $\left(p=7.19^{-14}\right)$, mid-calf level $(p=0.002)$, and mid-thigh level $(p=0.03)$ (Figure 3).

Furthermore, a post-study statistically significant difference was found between the 2 groups in mean values of the distance walked in 6MWT as a measure of functional capacity $(p=0.02)$, with a higher improvement in the MCDT group (Figure 4).

Table 1. Demographic data and baseline outcome measures in both groups

\begin{tabular}{|c|c|c|c|c|c|}
\hline \multicolumn{2}{|l|}{ Variables } & $\begin{array}{l}\text { MCDT group } \\
(\text { mean } \pm S D)\end{array}$ & $\begin{array}{l}\text { TPT group } \\
(\text { mean } \pm S D)\end{array}$ & $t$ & $p^{*}$ \\
\hline \multicolumn{2}{|l|}{ Age (years) } & $41.54 \pm 8.55$ & $43.28 \pm 8.29$ & 0.52 & $0.47^{* *}$ \\
\hline \multicolumn{2}{|l|}{ Height (m) } & $1.66 \pm 0.06$ & $1.67 \pm 0.05$ & 0.42 & $0.52^{* *}$ \\
\hline \multicolumn{2}{|l|}{ Weight (kg) } & $84.58 \pm 4.22$ & $86.44 \pm 4.9$ & 2.01 & $0.16^{* *}$ \\
\hline \multicolumn{2}{|c|}{ Body mass index $\left(\mathrm{kg} / \mathrm{m}^{2}\right)$} & $30.65 \pm 2.1$ & $30.9 \pm 1.87$ & 0.2 & $0.65^{* *}$ \\
\hline \multirow{4}{*}{ Oedema girth $(\mathrm{cm})$} & Foot $\left(5^{\text {th }}\right.$ metatarsal head $)$ & $29.75 \pm 2.63$ & $30.15 \pm 3.22$ & 0.22 & $0.64^{\star *}$ \\
\hline & Medial malleolus & $40.28 \pm 9$ & $39.76 \pm 9.53$ & 0.38 & $0.85^{\star *}$ \\
\hline & $20 \mathrm{~cm}$ below patella & $54.1 \pm 5.54$ & $54.06 \pm 7.78$ & 0.01 & $0.97^{* *}$ \\
\hline & $20 \mathrm{~cm}$ above patella & $89.15 \pm 10.02$ & $90.4 \pm 6.58$ & 0.27 & $0.61^{* *}$ \\
\hline \multirow{4}{*}{ Skin thickness (mm) } & Foot & $0.60 \pm 0.052$ & $0.60 \pm 0.06$ & 0.01 & $0.93^{* *}$ \\
\hline & Medial malleolus & $0.33 \pm 0.08$ & $0.33 \pm 0.06$ & $7.1^{-6}$ & $0.99^{* *}$ \\
\hline & Mid-calf & $0.34 \pm 0.04$ & $0.33 \pm 0.05$ & 0.15 & $0.7^{* *}$ \\
\hline & Mid-thigh & $0.35 \pm 0.06$ & $0.36 \pm 0.07$ & 0.15 & $0.7^{\star \star}$ \\
\hline \multicolumn{2}{|c|}{ 6-minute walking test $(\mathrm{m})$} & $398.25 \pm 30.7$ & $398.12 \pm 17.77$ & $3.3^{-4}$ & $0.99^{* *}$ \\
\hline
\end{tabular}

* level of significance at $p<0.05,{ }^{* *}$ non-significant

MCDT - modified complex decongestive therapy, TPT - traditional physical therapy

Table 2. Pre-post-test comparisons of oedema girth, skin thickness, and functional capacity within each group

\begin{tabular}{|c|c|c|c|c|c|c|c|}
\hline \multirow{2}{*}{\multicolumn{2}{|c|}{ Variables }} & \multicolumn{3}{|c|}{ MCDT group } & \multicolumn{3}{|c|}{ TPT group } \\
\hline & & \multirow{2}{*}{$\frac{t}{13.5}$} & \multirow{2}{*}{$\frac{p^{*}}{2.02^{-12 * *}}$} & \multirow{2}{*}{$\frac{\% \text { of change }}{19.2(\downarrow)}$} & \multirow{2}{*}{$\frac{t}{19.64}$} & \multirow{2}{*}{$\frac{p^{\star}}{2.69^{-16^{* \star}}}$} & \multirow{2}{*}{$\frac{\% \text { of change }}{13.47(\downarrow)}$} \\
\hline \multirow{4}{*}{ Oedema girth $(\mathrm{cm})$} & Foot ( $5^{\text {th }}$ metatarsal head $)$ & & & & & & \\
\hline & Medial malleolus & 9.03 & $5.05^{-9 * *}$ & $28.34(\downarrow)$ & 11.61 & $2.48^{-11 * *}$ & $15.27(\downarrow)$ \\
\hline & $20 \mathrm{~cm}$ below patella & 8.42 & $1.78^{-8 * *}$ & $16.57(\downarrow)$ & 14.3 & $3.07^{-13^{* *}}$ & $8.78(\downarrow)$ \\
\hline & $20 \mathrm{~cm}$ above patella & 36.47 & $7.4^{-22 \star \star}$ & $12.08(\downarrow)$ & 19.57 & $2.93^{-16^{* *}}$ & $7.15(\downarrow)$ \\
\hline \multirow{4}{*}{ Skin thickness (mm) } & Foot & 43.76 & $1.19^{-23 \star \star}$ & $70.32(\downarrow)$ & 33.48 & $1.17^{-21 * *}$ & $54.4(\downarrow)$ \\
\hline & Medial malleolus & 16.19 & $4.56^{-14 * *}$ & $63.32(\downarrow)$ & 16.24 & $1.92^{-14 * *}$ & $37.26(\downarrow)$ \\
\hline & Mid-calf & 11.86 & $2.8^{-11 * *}$ & $31.82(\downarrow)$ & 21.22 & $4.68^{-17 * *}$ & $21.02(\downarrow)$ \\
\hline & Mid-thigh & 5.27 & $2.4^{-5 \star \star}$ & $15.46(\downarrow)$ & 12.86 & $2.95^{-12 \star \star}$ & $9.12(\downarrow)$ \\
\hline \multicolumn{2}{|c|}{ 6-minute walking test $(\mathrm{m})$} & -13.15 & $3.49^{-12 * \star}$ & $9.84(\uparrow)$ & -22.38 & $1.38^{-17 * *}$ & $5.52(\uparrow)$ \\
\hline
\end{tabular}

* level of significance at $p<0.05,{ }^{* *}$ significant

$52 \downarrow$ - decrease, $\uparrow$ - increase, MCDT - modified complex decongestive therapy, TPT - traditional physical therapy 


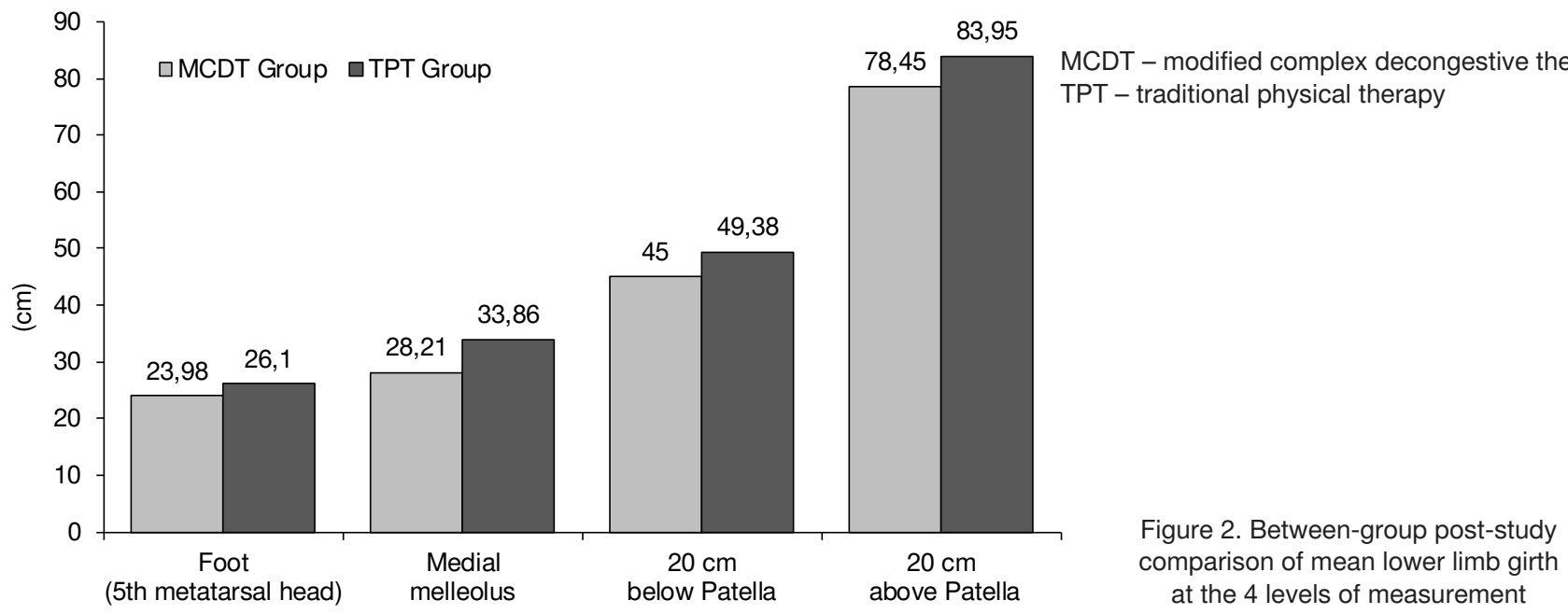

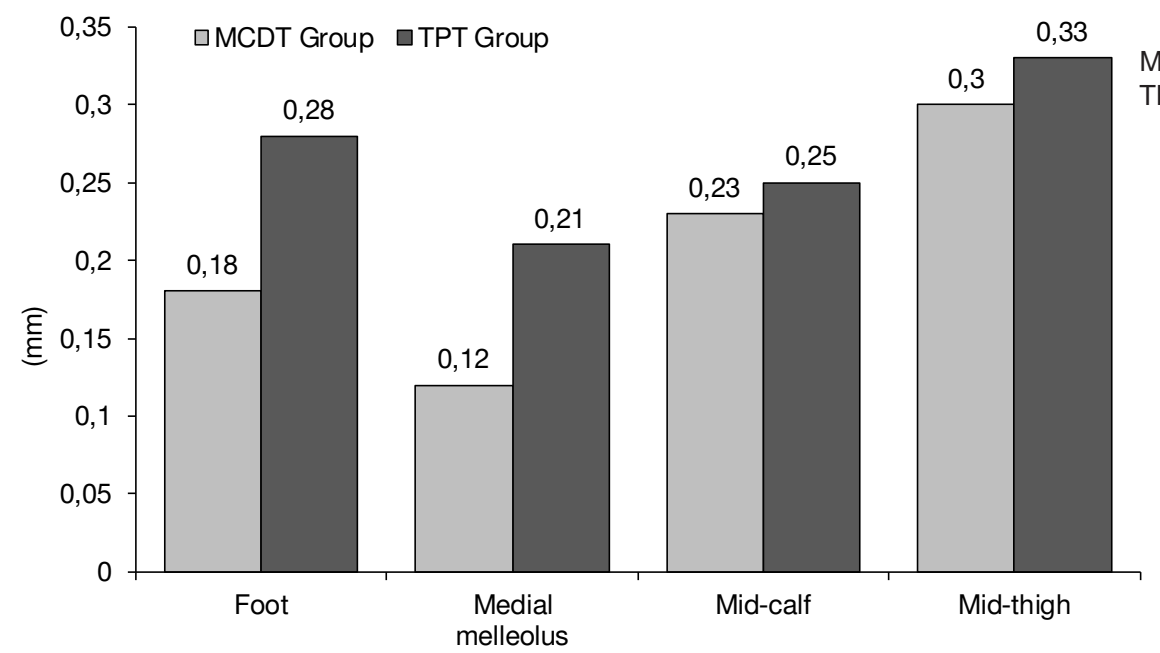

MCDT - modified complex decongestive therapy TPT - traditional physical therapy

Figure 3. Between-group post-study comparison of mean lower limb skin thickness at the 4 levels of measurement

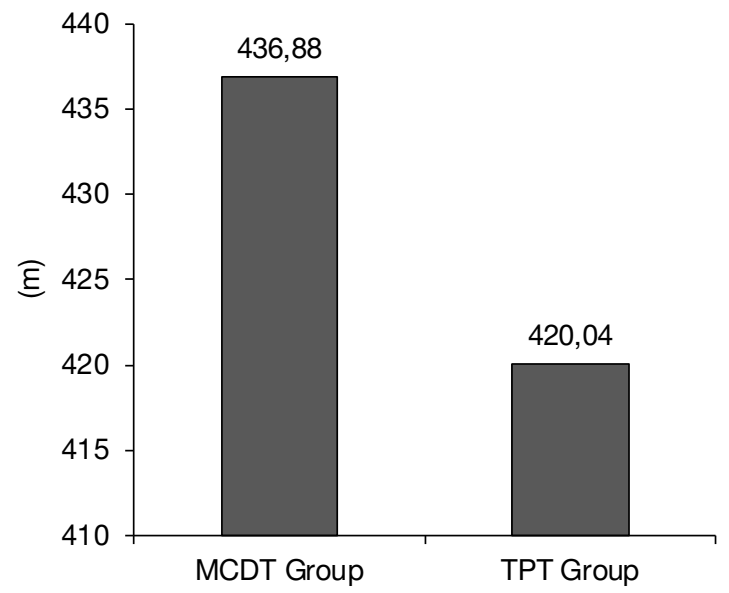

MCDT - modified complex decongestive therapy TPT - traditional physical therapy

Figure 4. Between-group post-study comparison of mean functional capacity

\section{Discussion}

Lymphoedema is a chronic debilitating disorder that requires prolonged and sustained treatment [7]. Although no definite cure has been yet discovered, many therapeutic options have been established to control the lymphoedema volume and prevent further fluid accumulation. Among these, CDT stands as the most important and commonly utilized approach to lymphoedema treatment [1].

The purpose of this study was to investigate the effect of an 8-week MCDT program on limb girth (oedema), skin thickness, and functional capacity in patients with LLSL in comparison with TPT. The results revealed that after 8 weeks of either MCDT or TPT, there were significant reductions in limb girth and skin thickness, as well as a significant increase in the functional capacity in the MCDT and TPT groups. But the comparison of the post-study results between the groups implied significant improvements in all outcome measures in favour of the MCDT group.

When dealing with lymphoedema, many barriers should be considered. The accumulated fluids are trapped either in fatty tissues that have a tendency to absorb the applied pressure without losing the constituents, or in fibrotic tissues that do not allow fluid displacement. Another problem is that the stagnated fluid is composed of water and lymph, which cannot be easily returned back to the venous system through damaged lymphatic vessels [10]. 
Although there are many available interventions for treatment of lymphoedema, CDT is a well-known conservative, multicomponent approach that aims to reduce limb girth. It is considered as the best among all known procedures because of its safety, no reported side effects, and cost-effective application [15]. A CDT program composed of MLD, skin care, compression, and exercises can reduce oedema and hence improve the functional activities [18]. Its effect can sustain over a long period, even for years [23-25].

The definite duration of a CDT program for patients with lower limb lymphoedema is not clearly established. There is a difference between the European model, recommending twice daily sessions for 4-6 weeks, and the North American model, recommending a single daily session for 3-4 weeks to gain significant achievements [26].

Previous studies point out that the effect of CDT on LLSL is time-dependent since more extended treatment programs provide more favourable results than short treatment programs. Daily CDT for an average of 13 days reduced lymphoedema by $68.1 \%$ [27], while daily CDT for 30 days in patients with unilateral LLSL reduced lymphoedema by $88 \%$ [23].

The results of the current study prove that MCDT can effectively reduce limb circumference in patients with LLSL, which can be attributed in part to the effect of MLD, which enhances and redirects the lymph flow and hence preserves lymphatic circulation [28]. MLD application prior to a compression garment can effectively reduce the amount of fluid proximal to the obstruction and improves the rate and efficacy of lymphatic fluid mobilization centrally towards the lymphatic duct as it forces the lymphatic fluid from the congested lower limb proximally towards the trunk and prevents damage of lymphatic capillaries [29].

MLD is superior in effect to traditional local massage. It enhances lymph fluid to flow more efficiently in lymph vessels towards better functioning vessels that drain in the nearest functioning quadrant of the body [30]. It preserves lymphatic function and prevents lymphatic trauma that can be caused by conventional massage [31].

MLD effect is further augmented with the application of a compressive garment that prevents re-accumulation of fluids and supports the normal lymph flow, especially during activities [23, 28]. A pressure garment effectively reduces the capillary filtration and enhances shifting of pooled fluid to the non-compressed proximal parts [32].

Seeking an alternative to multilayer bandaging for patients with lymphoedema becomes an essential requirement during treatment to eliminate complications of improper skin care and patients' non-adherence to bandaging [33]. The nonelastic containment LegAssistTH system was used instead of multilayer bandaging because of its ease of application, time saving, not interfering with patient's mobility or limiting their exercise performance, and no reported hazards [18].

The results of the current study agree with those obtained in previous research [27,34] which reported a significant reduction in limb girth in response to a program composed of MLD, compression therapy, skin care, and exercise training. Although the majority of researches recommend a daily CDT program [24, 26, 28, 35] for 4 weeks, a daily CDT program for about 2 weeks in patients with LLSL can reduce lymphoedema by about 67.7\% [12]. Moreover, Matthews and Smith [36] observed that twice-weekly sessions of temporary compression garments and MLD for only 1 week could effectively reduce LLSL.

The findings of this research prove that MCDT can effectively reduce skin thickness in patients with LLSL. This is in effectively reduced the thickness of skin and subcutaneous tissues in patients with lymphoedema. Skin thickness reduction in response to a CDT program was also emphasized by Yamamoto and Yamamoto [38], who found a significant effect of CDT on skin layer thickness in patients with lymphoedema.

The reduction in limb oedema and skin thickness reflects an improvement in the patients' abilities to perform their activities and hence in their functional performance.

\section{Limitations}

This study lacked follow-up and evaluation of long-term effects of MCDT and TPT in patients with LLSL.

\section{Conclusions}

The study revealed that both MCDT and TPT were effective approaches in the treatment of patients with LLSL. However, the application of MCDT yielded more significant effects than TPT on decreasing limb girth and skin thickness, as well as increasing functional capacity.

\section{Disclosure statement}

No author has any financial interest or received any financial benefit from this research.

\section{Conflict of interest}

The authors state no conflict of interest.

\section{Funding}

This study did not receive any form of funding.

\section{References}

1. International Society of Lymphology. The diagnosis and treatment of peripheral lymphedema: 2013 Consensus Document of the International Society of Lymphology. Lymphology. 2013;46(1):1-11.

2. Hassanein AH, Mulliken JB, Fishman SJ, Greene AK. Evaluation of terminology for vascular anomalies in current literature. Plast Reconstr Surg. 2011;127(1):347351; doi: 10.1097/PRS.0b013e3181f95b83.

3. Slavin SA. Secondary lymphedema. In: Greene AK, Slavin SA, Brorson H (eds.), Lymphedema. Cham: Springer; 2015; 79-95.

4. Damstra RJ, Partsch H. Prospective, randomized, controlled trial comparing the effectiveness of adjustable compression Velcro wraps versus inelastic multicomponent compression bandages in the initial treatment of leg lymphedema. J Vasc Surg Venous Lymphat Disord. 2013;1(1):13-19; doi: 10.1016/j.jvsv.2012.05.001.

5. Ferrandina G, Mantegna G, Petrillo M, Fuoco G, Venditti L, Terzano S, et al. Quality of life and emotional distress in early stage and locally advanced cervical cancer patients: a prospective, longitudinal study. Gynecol Oncol. 2012;124(3):389-394; doi: 10.1016/j.ygyno.2011. 09.041 .

6. Jensen MR, Simonsen L, Karlsmark T, Bülow J. Lymphoedema of the lower extremities - background, pathophysiology and diagnostic considerations. Clin Physiol Funct Imaging. 2010;30(6):389-398; doi: 10.1111/j. 1475-097X.2010.00969.x.

7. Rockson SG. Lymphedema. Vasc Med. 2016;21(1):7781; doi: 10.1177/1358863X15620852.

8. Bunce IH, Mirolo BR, Hennessy JM, Ward LC, Jones LC. Post-mastectomy lymphoedema treatment and mea- 
surement. Med J Aust. 1994;161(2):125-128; doi: 10.5694/j.1326-5377.1994.tb127342.x.

9. Moffatt CJ, Franks PJ, Doherty DC, Williams AF, Badger C, Jeffs E, et al. Lymphoedema: an underestimated health problem. QJM. 2003;96(10):731-738; doi: 10.1093/ qjmed/hcg126.

10. Badger C, Preston N, Seers K, Mortimer P. Physical therapies for reducing and controlling lymphoedema of the limbs. Cochrane Database Syst Rev. 2004;4:CD003141; doi: 10.1002/14651858.CD003141.pub2.

11. Gradalski T, Ochalek K, Kurpiewska J. Complex decongestive lymphatic therapy with or without Vodder II manual lymph drainage in more severe chronic postmastectomy upper limb lymphedema: a randomized noninferiority prospective study. J Pain Symptom Manage. 2015;50(6): 750-757; doi: 10.1016/j.jpainsymman.2015.06.017.

12. Ko DS, Lerner R, Klose G, Cosimi AB. Effective treatment of lymphedema of the extremities. Arch Surg. 1998; 133(4):452-458; doi: 10.1001/archsurg.133.4.452.

13. Lasinski BB. Complete decongestive therapy for treatment of lymphedema. Semin Oncol Nurs. 2013;29(1): 20-27; doi: 10.1016/j.soncn.2012.11.004.

14. Takeno Y, Arita H, Fujimoto E. Efficacy of complete decongestive therapy (CDT) on edematous rat limb after lymphadenectomy demonstrated by real time lymphatic fluid tracing. SpringerPlus. 2013;2(1):225; doi: 10.1186/ 2193-1801-2-225.

15. Mondry TE, Riffenburgh RH, Johnstone PAS. Prospective trial of complete decongestive therapy for upper extremity lymphedema after breast cancer therapy. Cancer J. 2004;10(1):42-48; doi: 10.1097/00130404-200401000 -00009 .

16. Leard T, Barrett C. Successful management of severe unilateral lower extremity lymphedema in an outpatient setting. Phys Ther. 2015;95(9):1295-1306; doi: 10.2522/ ptj.20140358.

17. Ferguson B. ACSM's guidelines for exercise testing and prescription, $9^{\text {th }}$ ed. J Can Chiropr Assoc. 2014;58(3): 328-328.

18. Holtgrefe KM. Twice-weekly complete decongestive physical therapy in the management of secondary lymphedema of the lower extremities. Phys Ther. 2006;86(8): 1128-1136; doi: 10.1093/ptj/86.8.1128.

19. Olsen LO, Takiwaki H, Serup J. High-frequency ultrasound characterization of normal skin. Skin thickness and echographic density of 22 anatomical sites. Skin Res Technol. 1995;1(2):74-80; doi:10.1111/j.1600-0846. 1995.tb00021.x.

20. Adedoyin RA, Adeyanju SA, Balogun MO, Akintomide AO, Adebayo RA, Akinwusi PO, et al. Assessment of exercise capacity in African patients with chronic heart failure using six minutes walk test. Int J Gen Med. 2010;3:109_ 113; doi: 10.2147/ijgm.s5533.

21. Földi M, Földi E. Guidelines for the application of MLD/ CDT for primary and secondary lymphedema and other selected pathologies. In: Földi M, Földi E, Strössenreuther RHK, Kubik S (eds.), Földi's textbook of lymphology for physicians and lymphedema therapists. München: Urban \& Fischer; 2006; 629-683.

22. King TI, Droessler JL. Physical properties of short-stretch compression bandages used to treat lymphedema. Am J Occup Ther. 2001;55(5):573-576; doi: 10.5014/ajot. 55.5.573.

23. Boris M, Weindorf S, Lasinski B, Boris G. Lymphedema reduction by noninvasive complex lymphedema therapy. Oncology. 1994;8(9):95-106.
24. Morgan RG, Casley-Smith JR, Mason MR, CasleySmith JR. Complex physical therapy for the lymphoedematous arm. J Hand Surg Br. 1992;17(4):437-441; doi: 10.1016/s0266-7681(05)80270-0.

25. Asdonk J. Effectiveness, indications and contraindications of manual lymph drainage therapy in painful edema [in German]. Z Lymphol. 1995;19(1):16-22.

26. Karadibak D, Yavuzsen T, Saydam S. Prospective trial of intensive decongestive physiotherapy for upper extremity lymphedema. J Surg Oncol. 2008;97(7):572-577; doi: 10.1002/jso.21035.

27. Liao S-F, Huang M-S, Li S-H, Chen I-R, Wei T-S, Kuo S-J, et al. Complex decongestive physiotherapy for patients with chronic cancer-associated lymphedema. J Formos Med Assoc. 2004;103(5):344-348.

28. Földi E. The treatment of lymphedema. Cancer. 1998; 83(12):2833-2834; doi: 10.1002/(sici)1097-0142 (19981215)83:12b+<2833::aid-cncr35>3.0.co;2-3.

29. Macdonald JM, Sims N, Mayrovitz HN. Lymphedema, lipedema, and the open wound: the role of compression therapy. Surg Clin North Am. 2003;83(3):639-658; doi: 10.1016/S0039-6109(02)00201-3.

30. Petrek JA, Pressman PI, Smith RA. Lymphedema: current issues in research and management. CA Cancer $\mathrm{J}$ Clin. 2000;50(5):292-307; doi: 10.3322/canjclin.50.5.292.

31. Eliska O, Eliskova M. Are peripheral lymphatics damaged by high pressure manual massage? Lymphology. 1995;28(1):21-30.

32. Partsch H, Damstra RJ, Mosti G. Dose finding for an optimal compression pressure to reduce chronic edema of the extremities. Int Angiol. 2011;30(6):527-533.

33. Randheer S, Kadambari D, Srinivasan K, Bhuvaneswari V, Bhanumathy M, Salaja R. Comprehensive decongestive therapy in postmastectomy lymphedema: an Indian perspective. Indian J Cancer. 2011;48(4):397-402; doi: 10.4103/0019-509X.92250.

34. Mahran SA, Moshref SS. The effectiveness of a modified complete decongestive therapy program in the treatment of lymphedema cases. JKAU Med Sci. 2011;18(4): 37-51; doi: 10.4197/med.18-4.4.

35. Weiss JM, Spray BJ. The effect of complete decongestive therapy on the quality of life of patients with peripheral lymphedema. Lymphology. 2002;35(2):46-58.

36. Matthews K, Smith J. Effectiveness of modified complex physical therapy for lymphoedema treatment. Aust J Physiother. 1996;42(4):323-328; doi: 10.1016/s00049514(14)60397-4.

37. Mellor RH, Bush NL, Stanton AWB, Bamber JC, Levick JR, Mortimer PS. Dual-frequency ultrasound examination of skin and subcutis thickness in breast cancerrelated lymphedema. Breast J. 2004;10(6):496-503; doi: 10.1111/j.1075-122X.2004.21458.x.

38. Yamamoto R, Yamamoto T. Effectiveness of the treatment-phase of two-phase complex decongestive physiotherapy for the treatment of extremity lymphedema. Int J Clin Oncol. 2007;12(6):463-468; doi: 10.1007/ s10147-007-0715-5. 